\section{Two novel germline mutations of the retinoblastoma gene $(R B 1)$ that show incomplete penetrance, one splice site and one missense}

EDITOR-Retinoblastoma (RB) is an intraocular eye tumour with a newborn incidence of 1 in 15 000-25 000. ${ }^{1}$ Of all retinoblastoma patients, $10-15 \%$ represent familial cases with an autosomal dominantly inherited predisposition for tumour development. On the basis of epidemiological data, Knudson ${ }^{2}$ hypothesised that retinoblastoma is a cancer caused by two mutational events. DNA analysis by Cavenee $e t a l^{\beta}$ confirmed this hypothesis. In the familial form, the first mutation is inherited via a germ cell and is, therefore, present in all somatic cells. The second mutation occurs in a somatic cell. In the non-hereditary form, both mutations occur in one and the same somatic cell. From this "two hit" model, it can be predicted that statistically a fraction of carriers of a mutation predisposing to retinoblastoma will never become affected. Indeed, in approximately $10 \%$ of families with hereditary retinoblastoma, obligate asymptomatic carriers of a mutant allele of the retinoblastoma gene (RB1) can be identified. ${ }^{1}$ Asymptomatic carriers of a predisposing $R B 1$ mutation are, however, not randomly distributed over families with hereditary retinoblastoma. Some families have been identified in which the majority of mutation carriers are either unaffected or only unilaterally affected. In a small number (fewer than $2 \%$ ) of mutation carriers, clinically benign retinal lesions, or "retinomas", occur. ${ }^{4}$ Most likely, retinoma arises when the second mutation occurs in an almost terminally differentiated retinal cell. ${ }^{5}$ Patients with retinoma can only be detected on ophthalmological inspection. They may, therefore, usually pass for being unaffected, but transmit the mutation predisposing to retinoblastoma to their offspring. Thus, they resemble true asymptomatic carriers of an RB1 germline mutation. DNA analysis of markers within and flanking the $R B 1$ gene allows the identification within retinoblastoma families of healthy subjects in whom the mutation is non-penetrant. ${ }^{6}$ It has been shown that the non-random distribution of asymptomatic carriers, the predominant occurrence of unilaterally affected subjects, and the high incidence of

Table 1 Primer sets used for multiplex PCR

\begin{tabular}{|c|c|c|c|c|}
\hline Reaction & Exon & Primer sequence & $\begin{array}{l}\text { Primer amount } \\
\text { (pmol/reaction) }\end{array}$ & $\begin{array}{l}\text { Product length } \\
(b p)\end{array}$ \\
\hline \multirow[t]{6}{*}{1} & \multirow[t]{2}{*}{4} & se 5'-GTGATTTGATGTAGAGCTGA-3' & 2 & \multirow[t]{2}{*}{422} \\
\hline & & as 5'-GGAAGCATTCAGAATGCATA-3' & 2 & \\
\hline & \multirow[t]{2}{*}{17} & se 5'-TTCCTATGAGTCCGTAGACT-3' & 5 & \multirow[t]{2}{*}{494} \\
\hline & & as 5'-GGGCTATAGACTGAAATAAC-3' & 5 & \\
\hline & \multirow{2}{*}{24} & se 5'-GGGTAGAGGTAACCTTTAAT-3' & 11 & \multirow{2}{*}{277} \\
\hline & & as 5'-ATTCTATGCAATATGCCTGG-3' & 11 & \\
\hline \multirow[t]{8}{*}{2} & \multirow[t]{2}{*}{9} & se 5'-TGCATTGTTCAAGAGTCAAG-3' & 9 & \multirow[t]{2}{*}{220} \\
\hline & & as 5'-GTTAGACAATTATCCTCCCT-3' & 9 & \\
\hline & \multirow[t]{2}{*}{10} & se 5'-TGTGTGCTGAGAGATGTAAT-3' & 3 & \multirow[t]{2}{*}{259} \\
\hline & & as 5'-AGGTCACTAAGCTAAAGACT-3' & 3 & \\
\hline & \multirow[t]{2}{*}{12} & se 5'-CCCTTCATTGCTTAACACAT-3' & 9 & \multirow{2}{*}{182} \\
\hline & & as 5'-CAAGTTTCTTTGCCAAGATA-3' & 9 & \\
\hline & \multirow[t]{2}{*}{13} & se 5'-ATTACACAGTATCCTCGACA-3' & 9 & \multirow[t]{2}{*}{236} \\
\hline & & as 5'-TATACGAACTGGAAAGATGC-3' & 9 & \\
\hline \multirow[t]{8}{*}{3} & \multirow{2}{*}{7} & se 5'-TATACTCTACCCTGCGATTT-3' & 6 & \multirow{2}{*}{238} \\
\hline & & as 5'-AAGCAACTGCTGAATGAGAA-3' & 6 & \\
\hline & \multirow[t]{2}{*}{15} & se 5'-CAATGCTGACACAAATAAGG-3' & 5 & \multirow[t]{2}{*}{225} \\
\hline & & as 5'-CGCACGCCAATAAAGACATA-3' & 5 & \\
\hline & \multirow[t]{2}{*}{26} & se 5'-AGTCATCGAAAGCATCATAG-3' & 5 & \multirow[t]{2}{*}{197} \\
\hline & & as 5'-AGACTTCTTGCAGTGTAAAC-3' & 5 & \\
\hline & 27 & se 5'-ACTTACCCAGTACCATCAAT-3' & 6 & 177 \\
\hline & & as 5'-TGAGAGACAATGAATCCAGA-3' & 6 & \\
\hline 4 & 3 & se 5'-TTCCAGGGAGGTTATATTCA-3' & 5 & 257 \\
\hline & & as 5'-TCACTATTTGGTCCAAGTTC-3' & 5 & \\
\hline & 11 & se 5'-CAACAGAAGCATTATACTGC-3' & 5 & 240 \\
\hline & & as 5'-CGTGAACAAATCTGAAACAC-3' & 5 & \\
\hline & 20 & se 5'-TTCTCTGGGGGAAAGAAAAGAGTGG-3' & 5 & 349 \\
\hline & & as 5'-AGTTAACAAGTAAGTAGGGAGGAGA-3' & 5 & \\
\hline & 25 & se 5'-CTAACTATGAAACACTGGCA-3' & 5 & 289 \\
\hline & & as 5'-AAATTGGTATAAGCCAGGAG-3' & 5 & \\
\hline 5 & 16 & se 5'-GGAAGAAGAACGATTATCCA-3' & 12 & 257 \\
\hline & & as 5'-CCAAAGAAACACACCACATT-3' & 12 & \\
\hline & 18 & se 5'-TTGGGAATTTCGAAGTAGAG-3' & 5 & 448 \\
\hline & & as 5'-CTTTATTTGGGTCATGTACC-3' & 5 & \\
\hline & 19 & se 5'-ATCTGTGATTCTTAGCCAAC-3' & 12 & 293 \\
\hline & & as 5'-CCCAGTAATGTACCTAGAAA-3' & 12 & \\
\hline & 22 & se 5'-ATATGTGCTTCTTACCAGTC-3' & 5 & 364 \\
\hline & & as 5'-GGTATTGGTGACAAGGTAGG-3' & 5 & \\
\hline 6 & 6 & se 5'-TGGACTGCATTCTATTATGC-3' & 5 & 279 \\
\hline & & as 5'-TAGTCCAAAGGAATGCCAAT-3' & 5 & \\
\hline & 8 & se 5'-CCATGCCTTCTCTTTGTATT-3' & 3 & 477 \\
\hline & & as 5'-TGTTAGGGAGAACTTACATC-3' & 3 & \\
\hline & 21 & se 5'-CAGTATGGAAAGAAATAACTC-3' & 5 & 344 \\
\hline & & as 5'-GTCAGACAGAATATATGATCTC-3' & 5 & \\
\hline & 23 & se 5'-ATCTAATGTAATGGGTCCAC-3' & 5 & 393 \\
\hline & & as 5'-GAGTTTCAAGAGTCTAGCTT-3' & 5 & \\
\hline
\end{tabular}


A

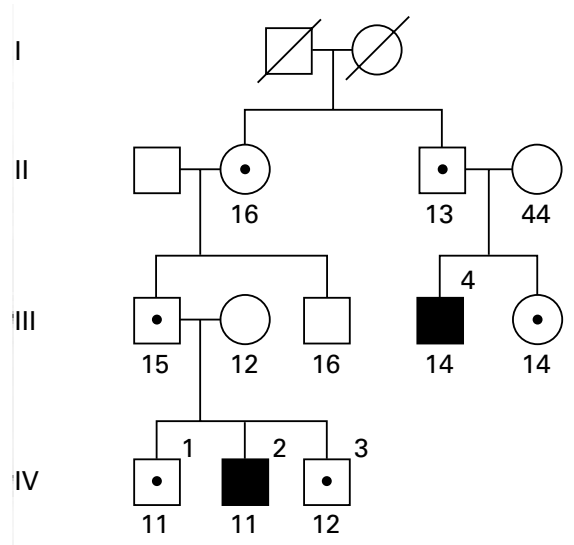

C

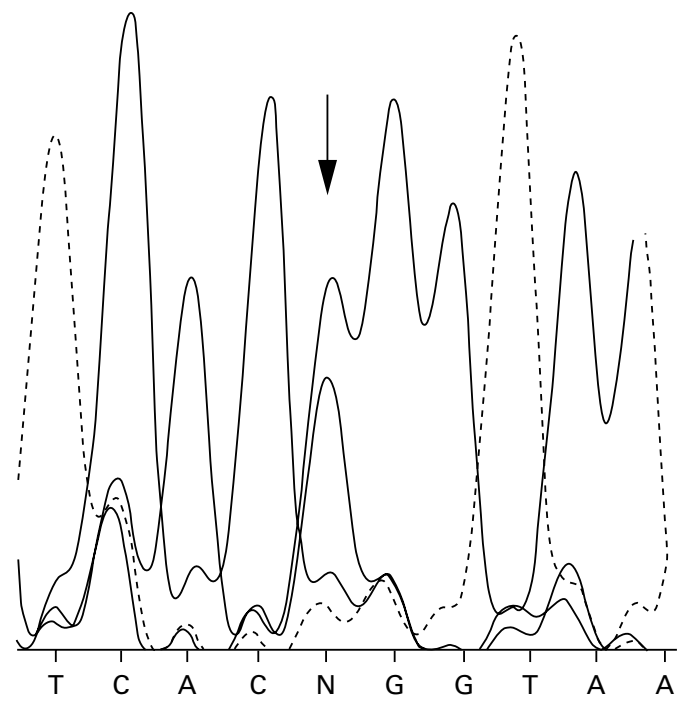

B
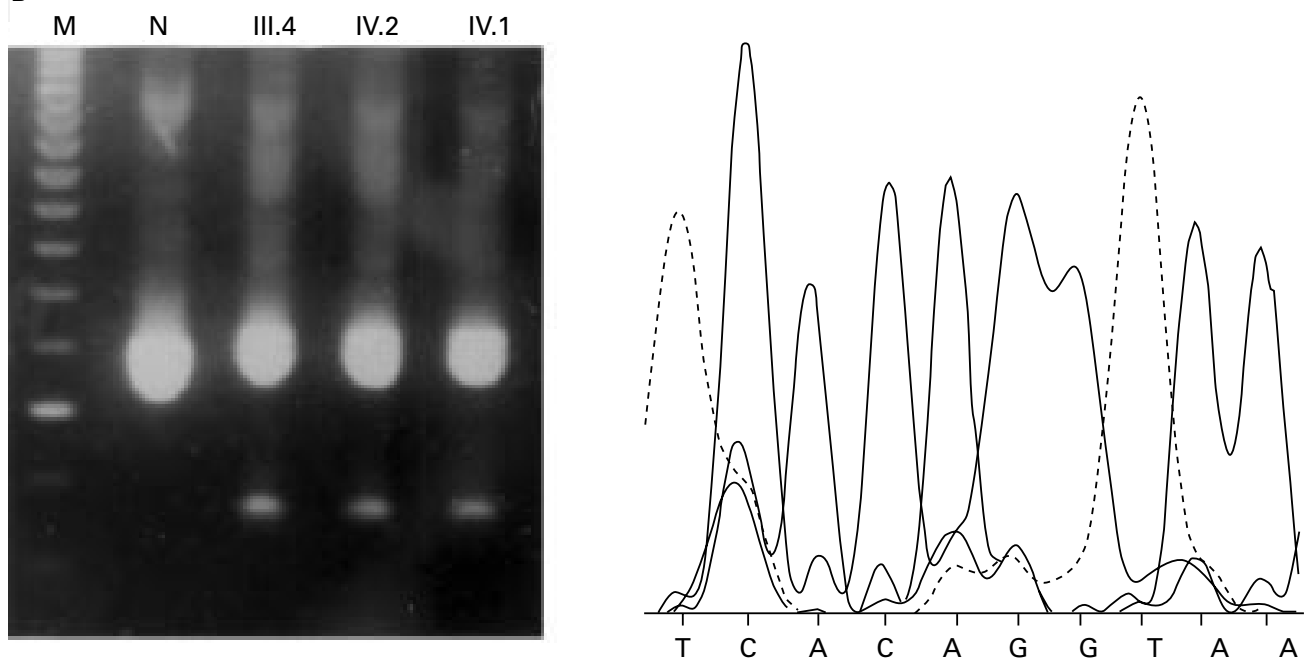

Figure 1 (A) Pedigree of family RB43. Affected subjects are indicated by black symbols, asymptomatic carriers by a dot. The segregation of the intragenic marker RB1.20 alleles is shown. (B) RT-PCR analysis of the RB1 transcript segment spanning exon 13. In the affected subjects III. 4 and IV.2, as well as in the asymptomatic carrier IV.1, an aberrant transcript is detected. The ratios of normal and aberrant transcript are identical in the affected subject compared with the asymptomatic subjects. The amount of aberrant transcript is severely reduced compared to the amount of normal transcript. A $50 \mathrm{bp}$ ladder is indicated by $M$ and a normal control by $N$. (C) Detection of the c.1331A>G mutation by direct sequencing of RB1 exon 13 PCR products. Normal and mutant sequences are shown. The heterozygous $A / G$ nucleotide is indicated by an arrow and an $N$.

retinomas in particular families is caused by partially defective $R B 1$ alleles because of mutations in the promoter region, ${ }^{78}$ particular missense mutations, ${ }^{9}$ a genomic in frame deletion, ${ }^{10}$ a large genomic deletion encompassing $R B 1$ exons 24 and $25,{ }^{11}$ or a splice site mutation leading to in frame exon skipping. ${ }^{12}$ From our own mutation screening in retinoblastoma families, we present two novel specific mutations apparently associated with incomplete penetrance.

One of the families described was referred to us directly, the other by the Departments of Clinical Genetics and Ophthalmology, Free University Hospital, Amsterdam. DNA and RNA were isolated from peripheral blood using standard methods. The analysis of the marker RB1.20 was performed as previously described. ${ }^{13}$

For initial mutation scanning, six multiplex PCR reactions were performed. In each reaction, three or four $R B 1$ exons were amplified simultaneously. In order to obtain PCR bands of the same intensity, it is often necessary to vary the amount of primer in a reaction, since some primers amplify better than others under standard conditions. Optimised primer sequences, amounts of primer per reaction, and product lengths are listed in table 1. For control experiments and sequencing, each exon was amplified separately using the same reaction conditions. Using a primer design program (Scientific and Educational Software, Durham, USA), primers located in the flanking introns were selected from the human retinoblastoma sequence. ${ }^{14}$ Amplification of genomic DNA was performed as previously described with the addition of $\mathrm{MgCl}_{2}$ to a final concentration of $3.0 \mathrm{mmol} / 1$ to reaction 5 .

The multiplex PCR products were analysed by means of the SSCP technique. To improve the resolution of the SSCP analysis, restriction cleavage of reactions 1,5 , and 6 was carried out with RsaI, PstI, and TaqI, respectively. To increase the detection rate of SSCP variant bands, each set of reactions was analysed using three gel conditions: (1) denaturing $6 \%(\mathrm{w} / \mathrm{v})$ polyacrylamide gel $(19 / 1(\mathrm{w} / \mathrm{w})$ acrylamide/bisacrylamide) containing $7 \%(\mathrm{w} / \mathrm{v})$ urea for analysis of small length alterations, (2) non-denaturing $6 \%$ 
$(\mathrm{w} / \mathrm{v})$ polyacrylamide gel $(19 / 1 \quad(\mathrm{w} / \mathrm{w})$ acrylamide/ bisacrylamide) containing $10 \%(\mathrm{v} / \mathrm{v})$ glycerol, and (3) $0.5 \times$ MDE gel (FMC-Bioproducts, Rockland, Maine, USA), according to the manufacturer's recommendations. Samples were denatured and electrophoresed for four hours at $20^{\circ} \mathrm{C}$ and $2000 \mathrm{~V}$ in a Macrophor electrophoresis apparatus (Pharmacia, Uppsala, Sweden) at room temperature. After electrophoresis, gels were dried and exposed to $x$ ray film. $R B 1$ exon 14 was analysed by direct sequencing.

RNA was isolated using the RNAZOL-B kit (Tel-Test Inc, Friendswood, Texas, USA). Subsequently, cDNA was synthesised with the Ready To Go first strand beads (Pharmacia, Uppsala, Sweden) by using a specific 3' primer: 5'-CAGACAGAAGGCGTTCACAA-3'. RT-PCR was performed using this primer and the 5'-primer: 5'GAAACACAGAGAACACCACG-3'. Nested PCR was performed using a primer located in exon 11 and a primer located in exon 15 of the RB1 gene: 5'-CCAGTT AGGACTGTTATGAA-3' and 5'-GGATAATCGTTC TTCTTCTG-3'. PCR products were analysed on an agarose gel.

Fragments amplified by PCR showing an aberrant SSCP pattern were subjected to direct sequencing by an automated sequencer (ALF, Pharmacia, Uppsala, Sweden). Reverse primers were extended by an M13 sequence (CGACGTTGTAAAACGACGGCCAGT) to enable the use of an identical M13 primer in all sequence reactions.

In the low penetrance family RB43, shown in fig $1 \mathrm{~A}$, a splice donor site mutation was identified. An A to G transition occurred at the last but one nucleotide of exon 13 (c.1331A $>\mathrm{G}$ mutation; cf sequence analysis in fig 1C), altering the splice donor site. By RT-PCR and subsequent sequencing we could show that this mutation results in loss of exon 13 in the RB1 mRNA. Skipping of exon 13, which consists of 117 nucleotides, does not alter the reading frame. In addition to the two unilaterally affected subjects in this pedigree, six asymptomatic carriers of the c. $1331 \mathrm{~A}>\mathrm{G}$ mutation were identified. Both the affected subjects and the six asymptomatic carriers shared an identical allele of the intragenic marker RB1.20 (fig 1A). Ophthalmological investigation did not show any indication of retinoma, nor was there any indication of a second primary tumour in any of these subjects. A similar mutation (c.1332G $>$ A, a $G$ to A transition of the last nucleotide of this exon) occurred in the non-familial bilateral case RB163, also leading to loss of exon 13 from the aberrant $R B 1$ transcript (data not shown). The ratios of the amount of normal length and aberrant length RT-PCR products from transcripts were identical for both mutations (data not shown), as well as the ratios of normal length and aberrant length RT-PCR products from transcripts in affected subjects compared to asymptomatic carriers in family RB43 (fig 1B). Apparently, more normal than aberrant transcript was produced, raising the possibility that the aberrant transcript is unstable. Since no other family members could be studied in family RB163, we do not know whether this mutation was already asymptomatically present in one of the parents, nor can we estimate the degree of penetrance in this family.

In family RB-K, one of the incomplete penetrance families in which we had previously identified an asymptomatic carrier by linkage analysis, ${ }^{6}$ a missense mutation in exon 17 was identified (fig 2). Codon 563 for tryptophan changes into a leucine by a $\mathrm{G}$ to $\mathrm{T}$ transversion (W563L). The mutation abolishes an NlaIII site. The mutation was present in all bilaterally affected subjects as well as the asymptomatic subject previously identified by linkage analysis (fig 2). In the other incomplete penetrance retinoblastoma family previously described, RB-E, sequence analysis of the complete $R B 1$ coding region including the exon/intron junctions (except exon 1) failed to detect a mutation in the coding sequence and flanking intronic sequences. Most likely, in this family, there is a mutation in $R B 1$ exon 1, in the promoter region or in an intronic sequence that has not been analysed.

In the low penetrance family RB43, a splice donor site mutation c.1331 $\mathrm{A}>\mathrm{G}$ was identified, leading to loss of exon 13 in the RB1 mRNA. Because exon 13 consists of 117 nucleotides and hence the reading frame of the coding sequence remains intact in the mutant $R B 1$ allele segregat-

A
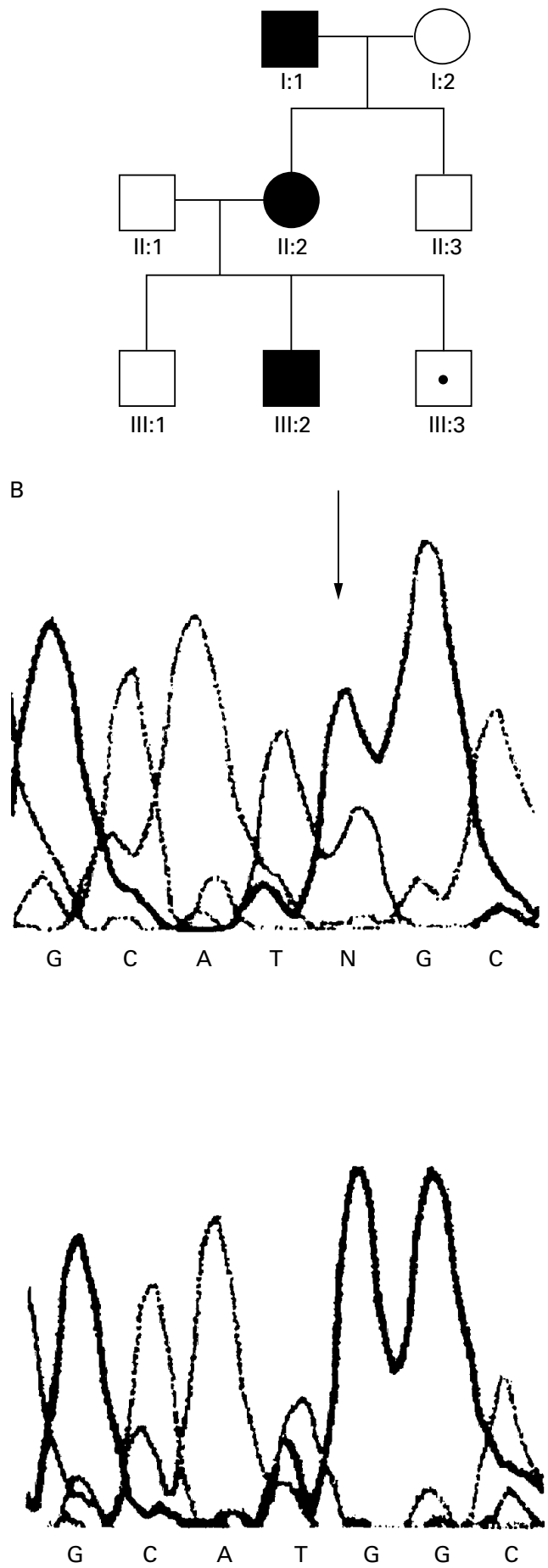

Figure 2(A) Pedigree of family RB-K. Affected subjects are indicated by black symbols, the asymptomatic carrier by a dot. (B) Detection of the W563L by direct sequencing of RB1 exon 17 PCR products. Normal and mutant sequences are shown. The heterozygous $T / G$ nucleotide is indicated by an arrow and an $N$. 
ing in this family, we hypothesise that the lack of $R B 1$ coded for by exon 13 has only a mild effect on cellular function of the RB protein. This mutation resembles the low penetrance mutation identified by Schubert et al, ${ }^{12}$ which causes skipping of exon 21 from the $R B 1$ transcript owing to a $\mathrm{G}$ to $\mathrm{A}$ transition at the last nucleotide of this exon. This mutation did not cause a frameshift either. The reduced amount of aberrant transcript is probably caused by its instability.

The cellular effect of splice site mutations has to be evaluated carefully. Not all splice site mutations result in a shift of the reading frame. Consequently, they may result in a less severe clinical presentation or even lack clinical symptoms in a proportion of mutation carriers in a particular family. Such a pedigree would thus present with incomplete penetrance. In some cases it is difficult to predict what the effect of a splice site mutation will be at the mRNA level, that is, which exons will be skipped, or whether alternative cryptic splice sites will be used. Ideally, the effect of any splice site mutation should be studied at the mRNA level.

The W563L mutation detected in family RB-K represents a missense mutation. At the protein level, this mutation is located within the oncogenic binding pocket domain $\mathrm{A}$, a region of the retinoblastoma protein with binding capacity for the SV40 large $\mathrm{T}$ antigen or the adenovirus $\mathrm{E} 1 \mathrm{~A}$ protein. Out of 74 different $R B 1$ mutations identified in a cohort of familial/bilateral retinoblastoma patients (initially identified by SSCP, later by an updated DGGE approach; manuscript in preparation), only four are missense mutations, including the W563L mutation and also a $\mathrm{R} 661 \mathrm{~W}$ mutation, previously also described in a reduced penetrance family. ${ }^{9}$ The other two missense mutations identified in our cohort were an L657P mutation in a familial case, without any indication of reduced penetrance in the pedigree, and an M704R mutation in a sporadic bilateral case. From this we conclude that in families in which $R B 1$ missense mutations segregate, the likelihood of the occurrence of asymptomatic carriers may be increased, dependent, however, on the particular mutation identified.

We would like to thank the retinoblastoma families and their clinicians for collaboration in this study.
H SCHEFFER* P VAN DER VLIES* $M$ BURTON $^{\star}$ E VERLIND* A C MOLL† $S$ M IMHOF† C H C M BUYS*

*Department of Medical Genetics, University of Groningen, Antonius Deusinglaan 4, NL-9713 AW Groningen, The Netherlands

†Department of Ophthalmology, Free University Hospital, De Boelelaan 1117, NL-1081 HV Amsterdam, The Netherlands

Correspondence to: Dr Scheffer, h.scheffer@medgen.azg.nl

1 Vogel F. Genetics of retinoblastoma. Hum Genet 1979;52:1-54

2 Knudson AG Jr. Mutation and cancer: statistical study of retinoblastoma. Proc Natl Acad Sci USA 1971;68:820-3.

3 Cavenee WK, Dryja TP, Phillips RA, Benedict WF, Godbout R, Gallie BL, Murphree AL, Strong LC, White R. Expression of recessive alleles by chromosomal mechanisms in retinoblastoma. Nature 1983;305:779-84

4 Connolly MJ, Payne RH, Johnson G, Gallie BL, Allderdice PW, Marshall WH, Lawton RD. Familial, EsD-linked, retinoblastoma with reduced penetrance and variable expressivity. Hum Genet 1983;65:122-4.

5 Gallie BL, Squire JA, Goddard A, Dunn JM, Canton M, Hinton D, Zhu XP, Phillips RA. Mechanism of oncogenesis in retinoblastoma. Lab Invest 1990;62:394-408.

6 Scheffer H, te Meerman GJ, Kruize YC, van den Berg AH, Penninga DP, Tan KE, der Kinderen DJ, Buys CH. Linkage analysis of families with hereditary retinoblastoma: nonpenetrance of mutation, revealed by combined use of markers within and flanking the RB1 gene. Am f Hum Genet 1989;45:252-60.

7 Sakai T, Ohtani N, McGee TL, Robbins PD, Dryja TP. Oncogenic germ-line mutations in $\mathrm{Spl}$ and ATF sites in the human retinoblastoma gene. Nature 1991;353:83-6.

8 Cowell JK, Bia B, Akoulitchev A. A novel mutation in the promotor region in a family with a mild form of retinoblastoma indicates the location of a new regulatory domain for the RB1 gene. Oncogene 1996;12:431-6.

9 Onadim Z, Hogg A, Baird PN, Cowell JK. Oncogenic point mutations in exon 20 of the RB1 gene in families showing incomplete penetrance and
mild expression of the retinoblastoma phenotype. Proc Natl Acad Sci USA mild expression of

10 Dryja TP, Rapaport J, McGee TL, Nork TM, Schwartz TL. Molecular etiology of low-penetrance retinoblastoma in two pedigrees. Am f Hum Genet 1993;52:1122-8

11 Bremner R, Du DC, Connolly-Wilson MJ, Bridge P, Ahmad KF, Mostachfi H, Rushlow D, Dunn JM, Gallie BL. Deletion of RB exons 24 and 25 causes low-penetrance retinoblastoma. Am 7 Hum Genet 1997;61:556-70.

12 Schubert EL, Strong LC, Hansen MF. A splicing mutation in RB1 in low penetrance retinoblastoma. Hum Genet 1997;100:557-63.

13 Yandell DW, Dryja TP. Detection of DNA sequence polymorphisms by enzymatic amplification and direct genomic sequencing. Am f Hum Genet 1989;45:547-55

14 Toguchida J, McGee TL, Paterson JC, Eagle JR, Tucker S, Yandell DW, Dryja TP. Complete genomic sequence of the human retinoblastoma susceptibility gene. Genomics 1993;17:535-43. 